\title{
Two-level anterior cervical discectomy and cage-assisted fusion without plates
}

\author{
Roberto Assietti, M.D., Federica Beretta, M.D., and Cesare Arienta, M.D. \\ Division of Neurosurgery, Ospedale Fatebenefratelli and Oftalmico, Milano, Italy
}

\begin{abstract}
Object. Anterior cervical discectomy (ACD) is an effective and safe treatment for nerve root or spinal cord compression caused by disc herniation or spondylosis. Cervical interbody fusion allows preservation of the physiological lordosis and stability of the cervical spine. Based on data reported in the literature, fusion rates decrease significantly when more than one level undergoes surgery, and some authors recommend the addition of a plate system to improve results. At the authors' institution cervical carbon fiber cages (CFCs) are routinely used after ACD. They describe their experience in the treatment of 24 patients with two-level disease treated with CFCs alone.

Methods. Twenty-one patients with cervical radiculopathy and three with radiculomyelopathy underwent ACD. Surgery was performed at C5-6 and C6-7 in 18, at C4-5 and C5-6 in four, and at C3-4 and C5-6 in two patients. All the patients underwent magnetic resonance imaging and 15 also underwent computerized tomography (CT) to assess the results of surgery.

Radiculopathy improved after surgery in all the cases, whereas myelopathy resolved in only one patient. At 1 year fusion was achieved in $96 \%$ of the surgically treated discs; this was verified on cervical spine $\mathrm{x}$-ray films in all patients and on CT scans in three patients. Cervical lordosis was restored in eight of the nine patients in whom it was lost preoperatively. No complications related to cage extrusion and no cases of symptomatic pseudarthrosis were observed.

Conclusions. Interbody fusion cages have a load-sharing function and stabilize the spine to increase segmental stiffness, thus achieving fusion rates similar to those associated with bone grafts, even in multilevel disease.
\end{abstract}

\section{KEY WORDS • degenerative disc disease • anterior cervical discectomy • interbody fusion cage • cervical plate}

Anterior cervical discectomy has proven to be a safe and effective procedure for the treatment of degenerative disc disease. The anterior approach allows direct visualization of the entire interspace and wide decompression of the anterior aspect cervical spinal cord and nerve roots; it may be undertaken in cases of multilevel disease, and interbody fusion may be performed if required. ${ }^{18}$

Cloward $^{2}$ first described the anterior approach as an option for cervical disc herniation and cervical spondylosis in cases in which iliac crest bone graft fusion was performed. The Cloward fusion procedure has undergone several technical modifications and there is now no consensus regarding the best technique. The various advantages and the types of morbidity associated with the different procedures are still debated in the literature. . $^{3,11,13,16,19}$

Interbody fusion cages are hollow implants that restore physiological disc height, allowing bone growth within and around them, thus stimulating bone fusion. They have been developed to prevent disc space collapse and its relevant clinicoradiological consequences, as well as the do-

Abbreviations used in this paper: $\mathrm{ACD}=$ anterior cervical discectomy; $\mathrm{ACDF}=\mathrm{ACD}$ and fusion $; \mathrm{CFC}=$ carbon fiber cage; $\mathrm{CT}=$ computerized tomography; $\mathrm{MR}=$ magnetic resonance; $\mathrm{VB}=$ vertebral body. nor-site morbidity reported in conjunction with autologous bone graft procedures. ${ }^{1}$ The primary complications related to the implantation of fusion cages are subsidence into the adjacent VBs, cage dislocation, nonunion-related instability, and painful pseudarthrosis. ${ }^{22}$

According to the literature, fusion rates decrease significantly in multilevel surgery and some authors recommend the addition of a plate system to improve the results. 5,6,16

The purpose of this study was to assess the safety and effectiveness of carbon interbody fusion cages for cervical degenerative disc disease and their application in multilevel surgery without the additional use of an anterior plate system.

\section{CLINICAL MATERIAL AND METHODS}

\section{Patient Population}

Over a period of 3 years a total of 110 single- or multiple-level cervical discectomies were performed in our department. Twenty-four patients suffering from degenerative disease underwent two-level ACD and intersomatic fusion in which the Cornerstone CFC (Medtronic Sofamor Danek, Memphis, TN) was placed at both levels; no plate instrumentation was used. Fifteen patients were women 
and nine were men. The mean age was 43 years (range 35-60 years). Neurological signs at presentation are shown in Fig. 1. Eighteen patients suffered from moderate-to-severe neck pain preoperatively. Neck pain was graded using a 10-point visual analog scale with endpoint anchors of "no pain" and "severe pain." Comparison of pre- and postoperative pain status was performed using the Student t-test.

Indication for surgical treatment in our 24 cases was based on the following criteria: clinical evidence of degenerative multilevel disc disease, correlation between clinical symptoms/neurological signs and radiological findings, and failure of conservative therapeutic measures. In nine cases preoperative radiography demonstrated kyphotic deformity. Preoperatively all patients underwent MR imaging and 15 also underwent CT scanning.

Twenty-two patients underwent surgery at contiguous levels, whereas two underwent surgery at distant levels. A total of 48 levels were surgically treated (Fig. 2). Surgery was performed at C5-6 and C6-7 in 18 patients (Fig. 3), at C4-5 and C5-6 in four, and at C3-4 and C5-6 in two (Fig. 4). All patients underwent cervical radiography before discharge; they were fitted with a stiff cervical collar immediately after surgery and instructed to wear it continuously for 2 months. All the patients were followed clinically and radiologically for at least 1 year postoperatively.

\section{Criteria of Evaluation}

The following criteria were used to judge the success of surgery: recovery of neurological function, absence/presence and intensity of neck pain, extent of fusion on cervical x-rays films at 1 year, degree of spinal curvature curve on x-ray films at 1 year, positioning of the cage, and return to work.

\section{The Cornerstone Cage}

The hollow, trapezoidal Cornerstone cage (Fig. 5) is made of carbon fiber reinforced polymer, the elasticity of which is similar to cortical bone. It comes in five physiological heights: $4,5,6,7$, and $8 \mathrm{~mm}$, in a lordotic $\left(4^{\circ}\right)$ trapezoid design. The Cornerstone $\mathrm{CFC}$ is radiolucent: whereas the fusion mass can be visualized through the implant on traditional plain radiography, the x-ray markers render the implant itself visible. Because of the absence of metal, CT scans and MR images are free of distortion.

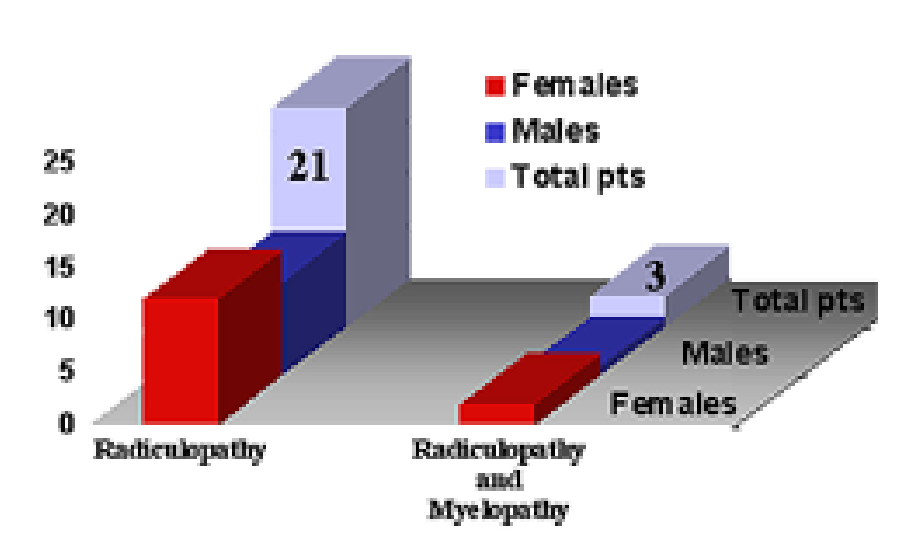

Fig. 1. Bar graph showing symptom distribution at presentation.

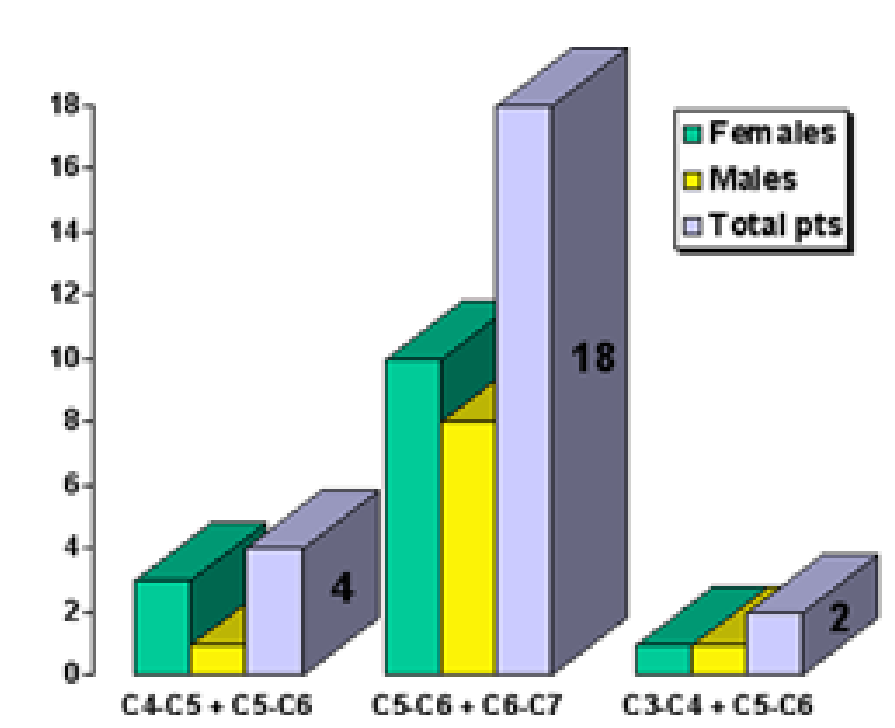

Fig. 2. Bar graph showing the distributions of surgically treated intervertebral disc levels.

The large opening offers maximum graft contact with the endplates, enhancing the fusion rate. The anterior semiopen configuration allows for postimplantation packing of bone graft. The rails created by a cutter intraoperatively help maintain the cage firmly in the appropriate position during motion. The cage is filled with bone chips

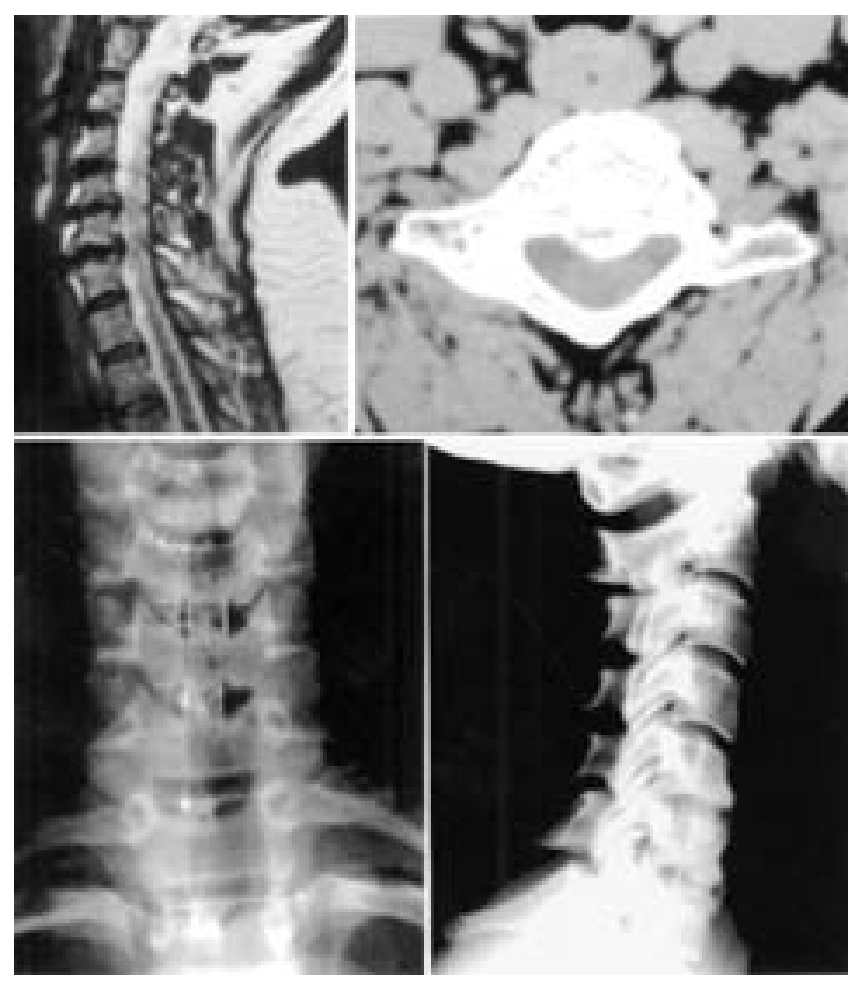

Fig. 3. Examples of $\mathrm{C} 5-6$ and C6-7 hard-disc herniations. Upper Left: Preoperative sagittal MR image. Upper Right: Axial CT scan obtained at C6-7, revealing a midline bone spur. Lower Left: Anteroposterior postoperative radiograph demonstrating cage position. Lower Right: Lateral postoperative radiograph. 


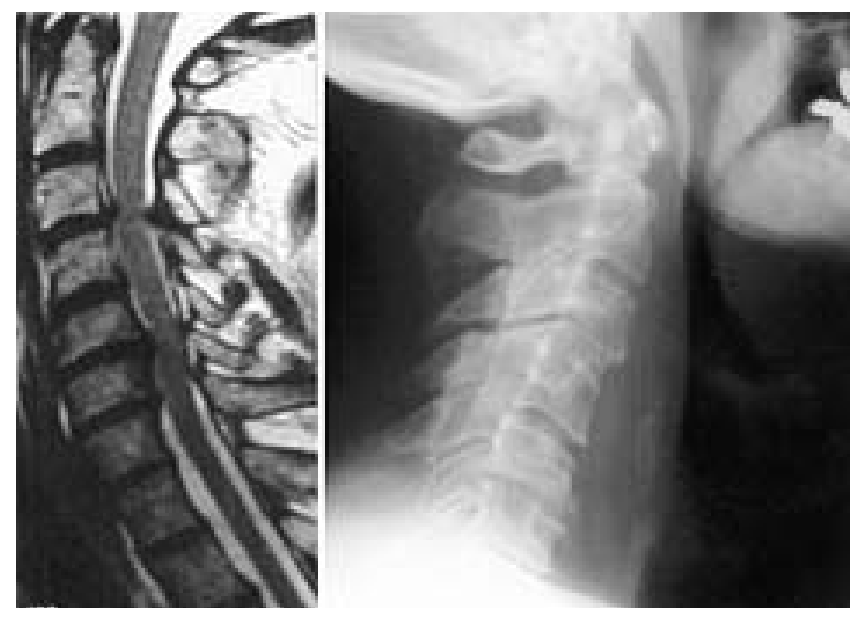

Fig. 4. Examples of C3-4 and C5-6 soft-disc herniations. Left: Preoperative sagittal MR image demonstrating a mild medullary lesion at the upper level. Right: Early lateral postoperative radiograph demonstrating settling of the lower cage.

obtained during the removal of the osteophytes or, when these are not available or are insufficient, with biphasic calcium phosphate (Medtronic Sofamor Danek).

\section{RESULTS}

\section{Recovery of Neurological Function}

All patients suffering from radiculopathy improved after surgery. Two patients still complained of moderate sensory loss at 6 months and one of mild radicular weakness. Of the three patients with radiculopathy and myelopathy, the radicular deficits resolved in all three whereas medullary function returned in only one.

\section{Neck Pain}

Postoperatively the mean visual analog pain score was 3 compared with a preoperative score of 7 ; the difference was statistically significant $(\mathrm{p}<0.01)$.

\section{Fusion Rate}

Each operative segment was deemed fused if a segmental motion of less than $2^{\circ}$ was observed on lateral flexionextension radiographs. Fusion was considered complete if the endplates had disappeared into both adjacent VBs and if the two VBs formed a block, with no radiolucency demonstrated except by the cage itself. At 1 year $46(96 \%)$ of the 48 surgically treated pateints showed complete radiographic fusion; in three cases, this was confirmed on follow-up CT studies (Fig. 6). The two patients in whom fusion was incomplete (Fig. 7) complained of mild neck pain but exhibited no symptoms of pseudarthrosis.

\section{Spinal Curvature}

The kyphotic deformity was corrected in eight of nine patients in whom it was lost preoperatively. No case of iatrogenic cervical deformity was observed postoperatively.

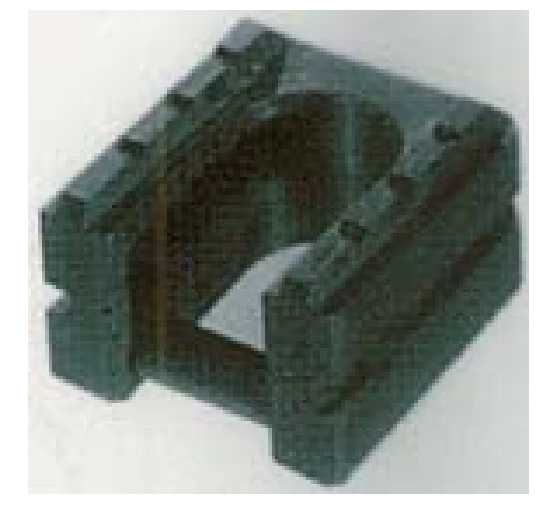

Fig. 5. Photograph of the Cornersone cage.

\section{Cage Positioning}

No case of cage extrusion was observed. In five (10.5\%) of 48 cases. We observed a settling of the cage inside the disc endplates we observed no evidence of symptom recurrence of or iatrogenic kyphosis any of these patients.

\section{Return to Work}

All patients who suffered preoperatively from radiculopathy improved after surgery and returned to their preoperative jobs, except for two patients with moderate radiculopathy who were obliged to change to occupations requiring milder activity. The two patients in whom medullary dysfunction did not improve after surgery had not returned to work at the 1-year follow up.

\section{Procedure-Related Complication}

There was no case of recurrent nerve palsy after surgery. One case of dysphagia was observed; however, this resolved in 2 weeks.

\section{DISCUSSION}

Although numerous technical variants of anterior cervical surgery can be used to achieve spinal cord and root decompression in patients with cervical radiculopathy and myelopathy, no single technique has been shown to be better or to produce superior results. In the literature, whether to use autologous bone grafts, allografts, bone substitutes, internal fixation, or any graft remains controversial. $2,7,12,14,16,19$

Some surgeons believe that anterior fusion after discectomy is not necessary. ${ }^{15,18,20}$ In a randomized study of onelevel anterior cervical surgery, Savolainen et al., ${ }^{16}$ reported that outcomes were similar for patients who underwent simple discectomy and those who underwent discectomy and fusion; they also found a higher incidence of kyphosis in patients in whom no instrumentation was placed. Wilson and Campbell ${ }^{23}$ reported good or excellent outcome after ACD alone in $85 \%$ of their series. Martins ${ }^{13}$ reported favorable outcome after ACD or ACDF in $92 \%$ of their patients in a 10-month follow-up period; significant kyphosis developed in $10 \%$ of the patients who underwent simple discectomy. Watters and Levinthal ${ }^{21}$ found that symptoms resolved earlier in patients who 


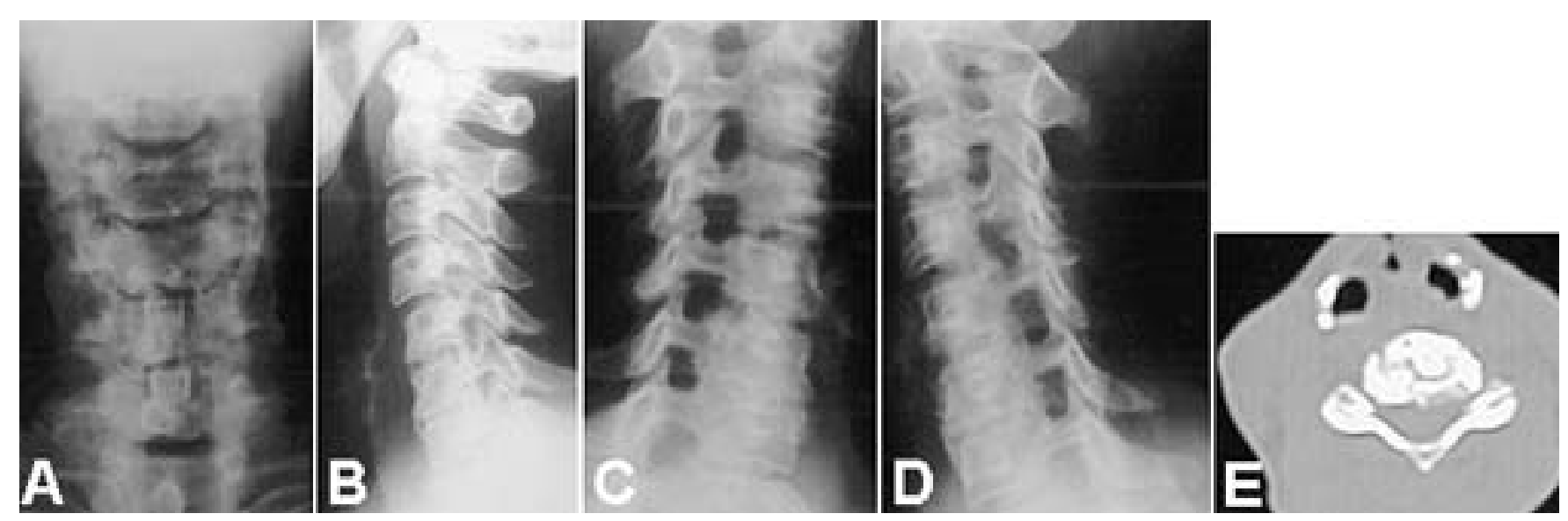

Fig. 6. Examples of C5-6 and C6-7 herniation at 1-year follow up. A: Anteroposterior radiograph. B: Lateral view radiograph. C and D: Oblique views of the same case. E: Axial CT (bone window) scan of the C5-6 level.

underwent ACDF compared with ACD; likewise, a higher rate of long-term success was demonstrated in the former group as well.

Sonntag, et al., ${ }^{18}$ have advocated ACD alone in most patients; fusion should be performed when instability occurs or may potentially occur because of an acute ruptured disc in conjunction with cervical VB fractures, extensive VB resection, or a previous or potential laminectomy. In their experience, however, ACD alone was usually associated with a longer period of postoperative neck and interscapular pain in the short term.

In our practice we perform cage-assisted fusion in all cases to avoid donor-site morbidity that is reportedly as high as $35 \%$. $^{1,17}$

The clinical consequences of postoperative foraminal narrowing and kyphosis are not well known; these conditions are often subclinical. Loss of sagittal-plane balance, however, is thought by some surgeons to accelerate adjacent-level degenerative disc disease. ${ }^{9}$

In two groups of patients who underwent fusion procedures, Hacker, et al., ${ }^{7}$ reported that postoperative sagittal malalignment was present in $19.8 \%$ of their patients in whom no abnormality was documented obvious abnormality before surgery. We observed mild postoperative kyphotic deformity in one case in which the cage subsided within the adjacent VB at 1-year follow up; no clinical consequences were observed. Cervical interbody fusion seems to allow preservation of the cervical physiological lordosis and its correction when it is lost preoperatively; it confers stability on the spine, promoting the fusion process. In our study the kyphotic deformity was corrected in eight of the nine patients in whom this abnormality was present preoperatively.

Based on reports in the literature, fusion rates decrease significantly when more than one level is surgically treated, and the rate of pseudarthrosis is higher in two-level compared with one-level surgery. ${ }^{24}$ Martin, et al., ${ }^{12}$ have reported a fusion rate of $90 \%$ when implanting freezedried allografts after one-level discectomy, which decreased to $72 \%$ after two-level fusions. In our study the fusion rate at 1 -year follow up was $96 \%$ in patients who underwent CFC-assisted two-level surgery; no cases of symptomatic pseudarthrosis were observed.
Some authors recommend the addition of a plate system to improve the results. In particular, they have reported that cervical plating-system increases fusion rates, allows earlier return to work, and limits subsidence and kyphotic deformity. ${ }^{4,6,16}$ Geer, et al., ${ }^{5}$ have observed greater incidences of kyphotic deformities, failed fusion, and subsidence in patients treated with ACD and iliac crest bone

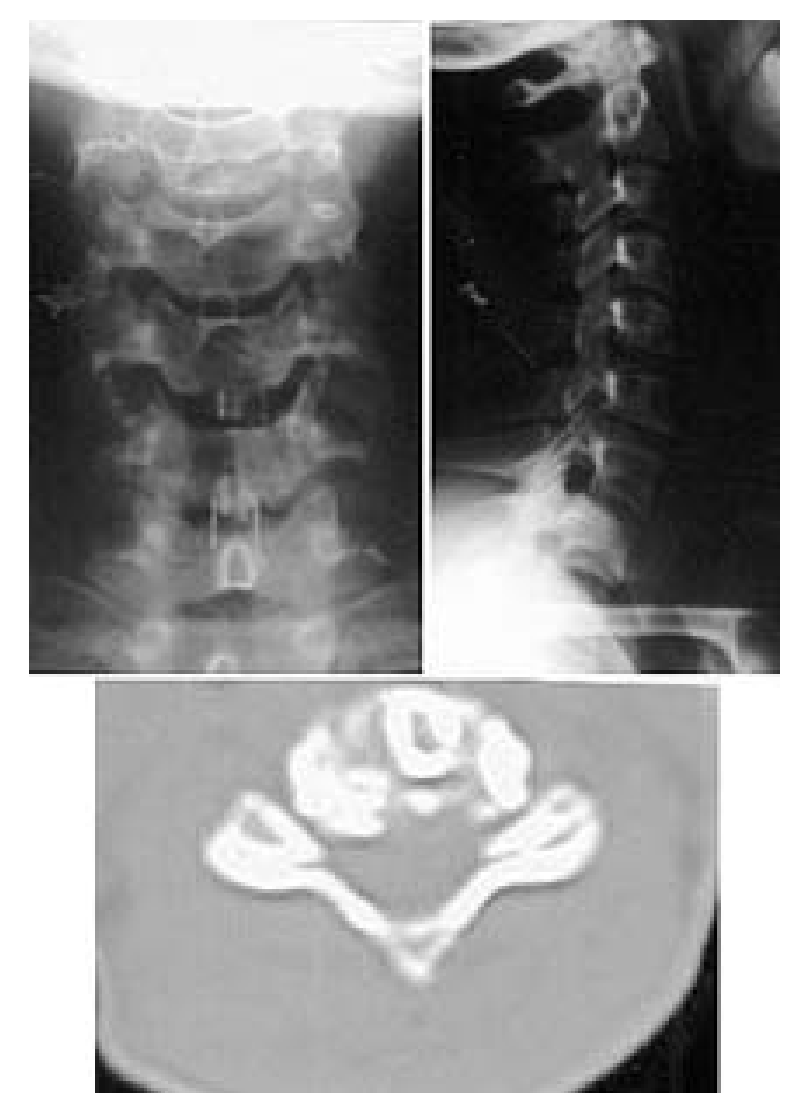

Fig. 7. Examples of C5-6 and C6-7 herniation at 9-month follow up without significant fusion. Upper Left: Anteroposterior radiograph. Upper Right: Lateral radiograph. Lower: Axial CT (bone window) scan of the C6-7 level. 
graft compared with allograft and fixation. They also reported that patients in whom a plating system was used returned to work sooner. Cervical plate fixation is not free from complications and morbidity, however, and there is a moderate risk of hardware failure. Lowery and McDonough $^{10}$ have reported a hardware failure rate of $35 \%$ in a series of 133 patients and a pseudarthrosis rate of $31 \%$ in index procedures, despite the fact that the hardware appeared intact. Esophageal injury is also more common when cervical plate fixation follows anterior cervical surgery; compared with plate fixation, cages are recessed below the margin of the VB, whereas even the lowest-profile plate system has contact with the overlying soft tissues. Cages are also cost effective. ${ }^{6}$ Savolainen, et al., ${ }^{16}$ reported a slightly better 6-month arthrodesis rate in patients who underwent plate-assisted fusion compared with those in whom plates were not used. At 4-year follow up, however, this difference had disappeared. Zdeblick and coworkers, ${ }^{24,25}$ found that, although the use of supplemental anterior internal fixation prevented graft extrusion, it failed to increase the histological union rate significantly in their goat ACDF model; the use of supplemental internal fixation did not reliably prevent graft collapse.

\section{CONCLUSIONS}

The ideal cervical fusion technique should guarantee $100 \%$ success in terms of fusion rates and neurological recovery, avoiding surgery-related complications. Cage constructs achieve immediate segmental internal fixation while providing structural support for the organic, fusionproducing bone material inside and around the cage itself, even at multiple levels. Our experience is preliminary and lacks historical and/or randmized controls. It is encouraging, however, and seems to demonstrate that cervical CFCs achieve high fusion rates, even in multilevel surgery; additionally, they seem to yield good or excellent clinical outcomes and minimal surgery- and device-related complications, even when anterior plates are not applied. Donor-site morbidity is completely avoided. A randomized trial is necessary to confirm our results.

\section{References}

1. Banwart JC, Asher MA, Hassanein RS: Iliac crest bone graft harvest donor site morbidity. A statistical evaluation. Spine 20: 1055-1060, 1995

2. Cloward RB: The anterior approach for removal of ruptured cervical disks. J Neurosurg 15:602-617, 1958

3. Fellrath RF Jr, Hanley EN Jr: Anterior cervical discectomy and arthrodesis for radiculopathy, in The Cervical Spine Research Society Editorial Committee (ed): The Cervical Spine, ed 3. Philadelphia: Lippincott-Raven, 1998, pp 785-798

4. Gaudinez RF, English GM, Gebhard JS, et al: Esophageal perforations after anterior cervical surgery. J Spinal Disord 13: $77-84,2000$

5. Geer C, Selden NRW, Papadopoulos SM: Anterior cervical plate fixation in the treatment of single-level cervical disc disease. J Neurosurg 90:410A, 1999 (Abstract)

6. Hacker RJ: A randomized prospective study of an anterior cervical interbody fusion device with minimum of 2 years of follow-up results. J Neurosurg 93 (Suppl 2):222-226, 2000
7. Hacker RJ, Cauthen JC, Gilbert TJ, et al: A prospective randomized multicenter clinical evaluation of an anterior cervical fusion cage. Spine 25:2646-2655, 2000

8. Helm GA, Dayoub H, Jane JA Jr: Bone graft substitutes for the promotion of spinal arthrodesis. Neurosurg Focus 10 (4): Article 4, 2001

9. Kettler A, Wilke HJ, Claes L: Effects of neck movements on stability and subsidence in cervical interbody fusion: an vitro study. J Neurosurg 94 (Suppl 1):97-107, 2001

10. Lowery GL, McDonough RF: The significance of hardware failure in anterior cervical plate fixation. Patients with 2- to 7-year follow-up. Spine 15:181-187, 1998

11. Lunsford LD, Bissonette DJ, Jannetta PJ, et al: Anterior surgery for cervical disc disease. Part 1: Treatment of lateral cervical disc herniation in 253 cases. J Neurosurg 53:1-11, 1980

12. Martin GJ Jr, Haid RW Jr, MacMillan M, et al: Anterior cervical discectomy with freeze-dried fibula allograft. Overview of 317 cases and literature review. Spine 24:852-859, 1999

13. Martins AN: Anterior cervical discectomy with and without interbody bone graft. J Neurosurg 44:290-295, 1976

14. Robinson RA, Walker AE, Ferlic DC, et al: The results of anterior interbody fusion of the cervical spine. J Bone Joint Surg Am 44:1569-1587, 1962

15. Rosenorn J, Hansen EB, Rosenorn MA: Anterior cervical discectomy with and without fusion. A prospective study. J Neurosurg 59:252-255, 1983

16. Savolainen S, Rinne J, Hernesniemi J: A prospective randomized study of anterior single-level cervical disc operations with long-term follow-up: surgical fusion is unnecessary. Neurosurgery 43:51-55, 1998

17. Sawin PD, Traynelis VC, Menezes AH: A comparative analysis of fusion rates and donor-site morbidity for autogenic rib and iliac crest bone grafts in posterior cervical fusions. J Neurosurg 88:255-265, 1998

18. Sonntag VKH, Klara P: Controversy in spine care. Is fusion necessary after anterior cervical discectomy? Spine 21: 1111-1113, 1996

19. Thalgott JS, Fritts K, Giuffre JM, et al: Anterior interbody fusion of the cervical spine with coralline hydroxyapatite. Spine 24:1295-1299, 1999

20. Thorell W, Cooper J, Hellbusch L, et al: The long-term clinical outcome of patients undergoing anterior cervical discectomy with and without intervertebral bone graft placement. Neurosurgery 43:268-274, 1998

21. Watters WC III, Levinthal R: Anterior cervical discectomy with and without fusion. Results, complications, and long-term follow-up. Spine 19:2343-2347, 1994

22. Wilke HJ, Kettler A, Goetz C, et al: Subsidence resulting from simulated postoperative neck movements: an in vitro investigation with a new cervical fusion cage. Spine 25:2762-2770, 2000

23. Wilson DH, Campbell DD: Anterior cervical discectomy without bone graft. Report of 71 cases. J Neurosurg 47:551-555, 1977

24. Zdeblick TA, Cooke ME, Kunz DN, et al: Anterior cervical discectomy and fusion using a porous hydroxyapatite bone graft substitute. Spine 19:2348-57, 1994

25. Zdeblick TA, Ghanayem AJ, Rapoff AJ, et al: Cervical interbody fusion cages. An animal model with and without bone morphogenetic protein. Spine 23:758-766, 1998

Manuscript received November 15, 2001

Accepted in final form December 19, 2001.

Address reprint requests to: Roberto Assietti, M.D., Division of Neurosurgery, Ospedale Fatebenefratelli and Oftalmico, Corso di Porta Nuova 23, 20123 Milano, Italy. email: assietti@tiscalinet.it. 